\title{
La métallisation de polymères : cuivre sur les fluoropolymères
}

\author{
E. Sacher, L. Martinu et M. Meunier
}

Groupe de Recherche en Physique et Technologie des Couches Minces (GCM), et, Département de Génie Physique, École Polytechnique de Montréal, CP. 6079, Succursale Centre-Ville, Montréal, Québec H3C 3A7, Canada

\begin{abstract}
Résumé : Une analyse des demandes des technologies ULSI et GSI indique la nécessité, dans les structures multicouches, de réduire le temps de délai du signal transmis entre les dispositifs à semi-conducteurs. Cette réduction est réalisée par l'utilisation d'un métal plus conducteur tel que le cuivre et un isolant moins capacitif que ceux utilisés actuellement, tel que les fluoropolymères. Dans cet article de revue, nous discutons brièvement nos résultats sur le dépôt de $\mathrm{Cu}$, par évaporation et par pulvérisation, sur les Teflons AF1600 et PFA, en particulier. Après une présentation des réactions interfaciales donnant lieu à l'adhérence, nous présentons une méthode pour améliorer cette adhérence consistant à faire une modification par plasma de la surface polymérique.
\end{abstract}

\section{INTRODUCTION}

Parmi les applications qui nécessitent la métallisation de polymères, la plus critique est sûrement dans le domaine des dispositifs multicouches microélectroniques formés de couches alternantes de polymère et de métal. Les couches métalliques sont gravées en forme de lignes et les couches polymériques servent d'isolant. Des "vias" sont aussi formés lorsque deux couches métalliques doivent être reliées. Ceci permet d'avoir plusieurs interconnexions électriques dans un petit volume, assurant ainsi que le temps de transmission entre les dispositifs à semi-conducteurs soit le plus court possible [1].

Le polymère doit subir des environnements hostiles lors de la fabrication, ce qui limite le choix à ceux qui sont résistants aux produits chimiques, à la température élevée et à l'humidité. Pour les technologies LSI (Large Scale Integration) et VLSI (Very Large Scale Integration), les polyimides et l'aluminium étaient les polymères et le métal de choix, respectivement. Cependant, pour les technologies ULSI (Ultra Large Scale Integration) et GSI (Giga Scale Integration), le temps de délai dû à l'interconnexion doit être réduit et de nouveaux matériaux doivent être utilisés. Le temps de délai, $\tau$, est le produit de la résistance, $\mathrm{R}$, le long de la ligne métallique que traverse le signal, et de la capacité, $\mathrm{C}$, de l'isolant entre ces lignes. Pour le réduire, on peut (1) réduire $\mathbf{R}$ par l'utilisation d'un métal dont la résistivité, $\rho$, est plus faible et (2) réduire $C$ par l'utilisation d'un isolant dont la permittivité, $k$, est plus faible. On peut, par exemple, réduire $\mathrm{C}$ de façon significative par le remplacement de polyimide $(\kappa \approx 3.4)$ par un fluoropolymère $(\kappa \approx 2.0)$. Également, on peut réduire $\mathrm{R}$ par le remplacement de l'aluminium utilisé présentement $(\rho=2.66 \mu \Omega \mathrm{m})$ par le cuivre $(\rho=1.67 \mu \Omega \mathrm{m})$. La substitution de l'un ou de l'autre réduit $\tau$ par $\sim 40 \%$. En utilisant des structures de cuivre et de fluoropolymères, le temps de délai pourrait être réduit par environ $60 \%$.

La métallisation des fluoropolymères par le cuivre présente un problème majeur au niveau de l'adhérence interfaciale [1]. Dans cet article, après une brève description des concepts généraux de l'adhérence entre le métal et le polymère, nous allons résumer les réactions d'interface entre le cuivre et le Teflon AF1600, choisi pour sa solubilité en solvants perfluorinés et, donc, susceptible à l'application par tournette. Par la suite, on couvrira brièvement le traitement du Teflon PFA par plasma afin d'améliorer l'adhérence entre le cuivre et le fluoropolymère.

\section{L'ADHÉRENCE ENTRE LE MÉTAL ET LE POLYMÈRE}

Le polymère et le métal ont des coefficients différents d'expansion thermique et hygroscopique. Ces différences sont suffisamment grandes pour que l'action de la fabrication même fasse introduire des 
contraintes internes élevées pouvant déchirer le dispositif. Cette destruction est évitée par une forte adhérence à chaque interface, maintenue à chaque étape lors de la fabrication, ainsi que lors de la vie d'utilisation du dispositif.

L'adhérence d'un polymère sur un métal présente des difficultés moins importantes que dans le cas d'un métal sur un polymère: en effet, si une réaction directe entre le polymère et les groupements réactifs à la surface métalliqué est difficile à effectuer, on a recours à l'utilisation de promoteurs d'adhérence. Ces derniers sont des molécules à deux groupements réactifs, l'un choisi pour sa grande réactivité à la surface du métal et l'autre, pour sa grande réactivité avec le polymère déposé là-dessus. Donc, le but de ce promoteur d'adhérence est de fournir une couche, entre le métal et le polymère, capable de réagir avec les deux. Les orthoesters de silane sont vendus commercialement à cette fin.

Par contre, pour le dépôt d'un métal sur le polymère, il n'existe pas de promoteurs d'adhérence utilisable dans ce cas. À sa place, il faut compter sur les réactions provoquées par l'énergie émise lors du dépôt du métal. Pour le dépôt par pulvérisation, le spectre du nombre de particules en fonction de l'énergie montre un maximum à une certaine énergie, suivi d'une longue queue vers les énergies plus hautes [2]. Pour le $\mathrm{Cu}$ pulvérisée par $\mathrm{Ar}^{+}$accéléré à $5 \mathrm{keV}$, le maximum est atteint à $\sim 950 \mathrm{~kJ} / \mathrm{mole}$ et l'énergie moyenne est de l'ordre de $3500 \mathrm{~kJ} / \mathrm{mole} \mathrm{[3].} \mathrm{En} \mathrm{comparant} \mathrm{ces} \mathrm{valeurs} \mathrm{aux} \mathrm{énergies} \mathrm{des} \mathrm{liens}$ covalents, $\sim 350-650 \mathrm{~kJ} / \mathrm{mole}[4,5]$, on conclut que la pulvérisation est capable de briser des liens et de provoquer des réactions interfaciales importantes. Dans le cas du dépôt par évaporation, l'énergie émise est celle de la condensation métallique, $\sim 290-500 \mathrm{~kJ} / \mathrm{mole}[6,7]$ pour les métaux utilisés. Étant à la même gamme d'énergie, ou plus faible, que les liens covalents, la provocation des réactions à la surface est attendue d'être moins importante. Les études de la métallisation des polymères, par XPS [8] et HREELS [9], qui utilisent les couches métalliques partielles, nous permettent de confirmer cette situation.

Parfois, particulièrement pour des polymères non-réactifs, les réactions provoquées n'amènent pas $a ̊$ une adhérence suffisamment élevée. Dans ces cas, on doit recourir aux modifications de la surface des polymères dans le but d'introduire les groupements souhaités.

\section{LA RÉACTION D'INTERFACE ENTRE LE CUIVRE ET LE TEFLON AF1600}

À l'aide de l'XPS, nous avons étudié les réactions d'interface entre le dépôt de cuivre obtenu par la pulvérisation ou l'évaporation et le substrat de Teflon AF1600 [10].

Le Teflon AF1600 est un copolymère formé de 35\% de tetrafluoropolymère (TFE) et de $65 \%$ de 2,2 bistrifluoromethyl-4,5difluoro-1,3dioxole (TDD); il a une unité de répétition de -(TFE)-(TDD) 2 . Des épaisseurs de 1 à $3 \mu \mathrm{m}$ sont obtenue selon la méthode recommandée par le fabricant, Dupont. Les dépôts de cuivre sont effectués par évaporation et par pulvérisation dans la chambre de préparation du système XPS à des taux de $2 \AA / \mathrm{s}$ et $4 \AA / \mathrm{min}$, respectivement. Les épaisseurs de cuivre varient entre $4 \AA \AA^{\circ}$ et 40 A.

Afin de tenir compte du déplacement dû à la charge durant l'exposition de l'échantillon aux rayons $\mathrm{X}$, le pic de $\mathrm{Cu} 2 \mathrm{p}_{3 / 2}$ pour l'échantillon le plus épais a été arbitrairement placé à $931 \mathrm{eV}$, qui se trouve à être l'énergie de liaison du cuivre métallique. Les spectres du $\mathrm{C} 1 \mathrm{~s}, \mathrm{~F} 1 \mathrm{~s}, \mathrm{O} 1 \mathrm{~s}$ et de $\mathrm{Cu} 2 \mathrm{p}_{3 / 2}$ ont été obtenues à différents temps d'évaporation et de pulvérisation. À la Figure 1, on montre certains de ces spectres et le Tableau 1 donne un résumé de notre analyse. Des nouveaux pics indiquent des changements chimiques dus à des réactions spécifiques entre le dépôt de cuivre et le Teflon AF1600. Pour l'évaporation, la concentration relative de carbure présent à la surface a été déterminée à partir du rapport entre la concentration totale de cuivre et la concentration de $\underline{\mathrm{C}}$ - $\mathrm{Cu}$ mesurée à partir du spectre de C1s. Ce rapport ne change essentiellement pas avec l'épaisseur, montrant qu'environ $50 \%$ du dépôt de cuivre reste dans l'état métallique. En général, les spectres XPS des dépôts par pulvérisation présentent les mêmes caractéristiques que ceux précédemment. Les positions des pics sont essentiellement identiques à $0.1 \mathrm{eV}$ près des dépôts par évaporation. Il y a par contre plusieurs nouveaux pics, indiquant la formation de nouvelles espèces de $\mathrm{Cu}-\mathrm{C}, \mathrm{Cu}^{+}$et $\mathrm{Cu}^{2+}$.

De plus, la Figure 1 résume schématiquement les principales réactions d'interface provoquées par l'évaporation et par la pulvérisation du cuivre sur le Teflon AF1600 déduites de l'analyse des spectres XPS. Pour l'évaporation, l'énergie de condensation du métal est la principale source d'énergie. Cette 
augmentation d'énergie à l'interface induit des réactions de dégradation, résultant en une perte uniforme de fluor à la surface du Teflon. Le cuivre se lie uniquement au carbone en formant des carbures dans tout le dépôt, dont la moitié reste dans l'état

Tableau 1. Résumé des nouveaux pics et des réactions de surface tels que déduits de l'analyse XPS. (d'après réf. 10, utilisé avec la permission d'Elsevier Science BV)

\begin{tabular}{|c|c|c|c|c|c|c|}
\hline & \multicolumn{3}{|c|}{ Évaporation $^{\mathrm{a}}$} & \multicolumn{3}{|c|}{ Pulvérisation $^{\mathrm{a}}$} \\
\hline & $\begin{array}{l}\text { nouveaux } \\
\text { pics } \\
(\mathrm{eV})\end{array}$ & attribution & réactions & $\begin{array}{l}\text { nouveaux } \\
\text { pics } \\
(\mathrm{eV})\end{array}$ & attribution & réactions \\
\hline C1s & $\begin{array}{l}283.7 \\
285.4 \\
287.3\end{array}$ & $\begin{array}{l}\underline{\mathrm{C}}-\mathrm{Cu} \\
\underline{\mathrm{C}}-\mathrm{C} \\
\underline{\mathrm{C}}-\mathrm{CF}[9]\end{array}$ & $\begin{array}{l}\text { formation de } \\
\text { carbure } \\
\text { graphitisation et } \\
\text { réticulation; } \\
\text { perte partielle } \\
\text { de F }\end{array}$ & $\begin{array}{l}283.7 \\
285.4 \\
287.3\end{array}$ & $\begin{array}{l}\text { C-Cu } \\
\text { C-C } \\
\underline{\mathrm{C}-\mathrm{CF}}\end{array}$ & $\begin{array}{l}\text { de carbure } \\
\text { graphitisation et } \\
\text { réticulation; } \\
\text { perte partielle de } \\
\text { F }\end{array}$ \\
\hline F1s & aucun & & $\begin{array}{l}\text { défluorination } \\
\text { uniforme }\end{array}$ & 682.5 & $\mathbf{F}$ & $\begin{array}{l}\text { défluorination } \\
\text { uniforme }\end{array}$ \\
\hline Ols & $\begin{array}{l}529.6 \\
530.4\end{array}$ & $\begin{array}{l}\text { deux sites o } \\
\text { légèrement } \\
\text { différents }\end{array}$ & $\begin{array}{l}\text { formation de } \\
\text { radicaux libres }\end{array}$ & $\begin{array}{l}529.6 \\
530.4\end{array}$ & $\begin{array}{l}\text { deux sites O } \\
\text { légèrement } \\
\text { différents }\end{array}$ & $\begin{array}{l}\text { formation de } \\
\text { radicaux libres; } \\
\text { perte de } \sim 30 \% \\
\text { de } O\end{array}$ \\
\hline $\mathrm{Cu} 2 \mathrm{p}_{3 / 2}$ & 931 & $\mathrm{C}-\underline{\mathrm{Cu}}$ et $\mathrm{Cu}^{0}$ & $\begin{array}{l}\mathrm{Cu}-\mathrm{C} \text { et } \mathrm{Cu} \\
\text { métallique } \\
\left(\mathrm{Cu}^{+} / \mathrm{Cu}^{0} \approx 1\right)\end{array}$ & $\begin{array}{l}932 \\
934.3 \\
936\end{array}$ & $\begin{array}{l}\mathrm{C}-\underline{\mathrm{Cu}} \\
\mathrm{Cu}^{+} \\
\mathrm{Cu}^{2+}\end{array}$ & $\begin{array}{l}\mathrm{Cu}-\mathrm{C} \\
\mathrm{CuF} \\
\mathrm{CuF}_{2} \\
\text { pas de } \mathrm{Cu}^{0}\end{array}$ \\
\hline
\end{tabular}

a. Effectué dans la chambre de préparation de l'XPS, et analysé sans exposition à l'atmosphère.
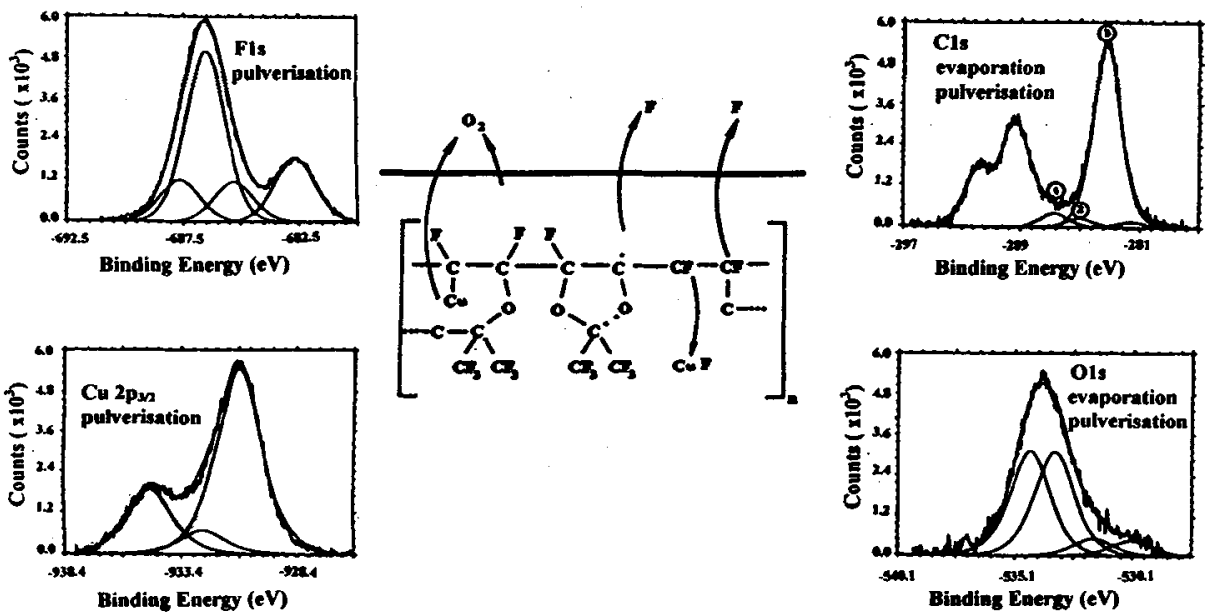

Figure 1: Spectres XPS témoins, obtenus après la métallisation par les méthodes indiquées, ainsi que les réactions provoquées dans le Teflon AF1600 déduites par leurs analyses. 
métallique. Il n'y a pas d'importante perte d'oxygène, bien que des modifications du spectre original du $\mathrm{O} 1 \mathrm{~s}$ indique un bris de lien d'oxygène du groupe dioxole pour produire des radicaux libres. Dans le cas de la pulvérisation, l'énergie cinétique des atomes de cuivre, qui est au moins un ordre de grandeur plus élevée que l'énergie de condensation relâchée par la condensation des atomes évaporés, donne de nouvelles réactions. Les atomes de $\mathrm{Cu}$ réagissent entièrement, en formant des carbures et des fluorures, tandis que le spectre du $\mathbf{C}$ indique des processus de dégradation semblables à ceux trouvés dans le cas de l'évaporation. L'énergie élevée cause non seulement des bris simples de liens pour produire des $-\mathrm{C}-\mathrm{O} \cdot$, mais des seconds bris, résultant en une perte d'atomes d'oxygène. De plus, quelques liens C-F sont brisés résultant en la formation de fluorure de cuivre.

\section{TRAITEMENT PAR PLASMA POUR L'AMÉLIORATION DE L’ADHÉRENCE}

Parmi les différentes méthodes pour améliorer l'adhérence aux polymères [traitement chimique (acides, bases), flammes, corona, ...], le traitement par plasma à basse pression attire beaucoup d'attention pour les raisons suivantes [11-14]: (i) le contrôle des interactions de plasma avec la surface permet de modifier toutes les caractéristiques associées avec tous les modèles d'adhérence $[15,16]$; b) le traitement est hautement uniforme, reproductible et il peut être optimisé pour la combinaison métal/polymère désirée; c) l'avancement des connaissances au niveau des sources pour générer le plasma, du diagnostic du plasma et de surface porte beaucoup de potentiel économique [14]; d) il n'y a pas de produits toxiques et les procédés plasma ne contaminent pas l'environnement.

Le domaine de modification des surfaces polymériques par plasma évolue très rapidement et plusieurs ouvrages de référence sont disponibles [11-14]. Dans le texte qui suit, nous décrivons brièvement les interactions de plasma avec les surfaces polymériques et montrons quelques exemples de contrôle de l'adhérence polymère/métal.

Le plasma est une source riche en particules énergétiques et chimiquement actives (électrons, ions, photons, radicaux), et le plasma interagit avec la surface polymérique typiquement via la chimie de radicaux libres $[17,18]$. Dans un plasma qui ne mène pas au dépôt d'une couche mince, quatre effets majeurs sont normalement observés. Chacun d'eux peut prédominer à un certain niveau selon la nature du gaz (chimie dans la phase gazeuse) et du substrat, du type de réacteur et des paramètres d'opération. Ces quatre effets sont [11]:

(i) nettoyage de surface, i.e. enlèvement de la contamination organique de la surface;

(ii) ablation, ou gravure, du matériau à la surface qui peut mener à l'enlèvement de la couche de faible adhérence et à l'augmentation de la superficie spécifique (rugosité);

(iii) réticulation ou branchement des molécules, ce qui peut augmenter la rigidité cohésive de la région près de la surface;

(iv) modification de la structure chimique qui peut se produire directement sous vide par l'action du plasma mais qui peut aussi être changée par l'exposition à l'atmosphère et une réaction subséquente avec l'oxygène atmosphérique et la vapeur d'eau.

Tous ces processus, séparément ou en synergie, ont un effet important sur les différents modèles de l'adhérence.

Les fluoropolymères possèdent une faible énergie de surface et une cohésion réduite. Ceci résulte typiquement en une mauvaise adhérence métallique. Dans les procédés plasma, on cherche des conditions optimisées de traitement qui nettoient la surface et qui enlèvent des liaisons fluorées en formant des sites pour la réaction subséquente avec le métal. Parmi les différents paramètres à optimiser lors d'un 
traitement plasma, les plus importants sont la nature du gaz, la densité de puissance et de la pression. Un exemple des effets de différents gaz sur la structure chimique à la surface du Teflon PFA [19] est illustré à la figure 2 par les spectres XPS C1s. On voit que la restructuration est la plus efficace après un traitement par $\mathrm{H}_{2}$, et elle est plus importante près de la surface qu'en profondeur. Evidemment, l'hydrogène peut réagir avec le fluor pour former du HF volatile, ce qui résulte en une dégradation du polymère.

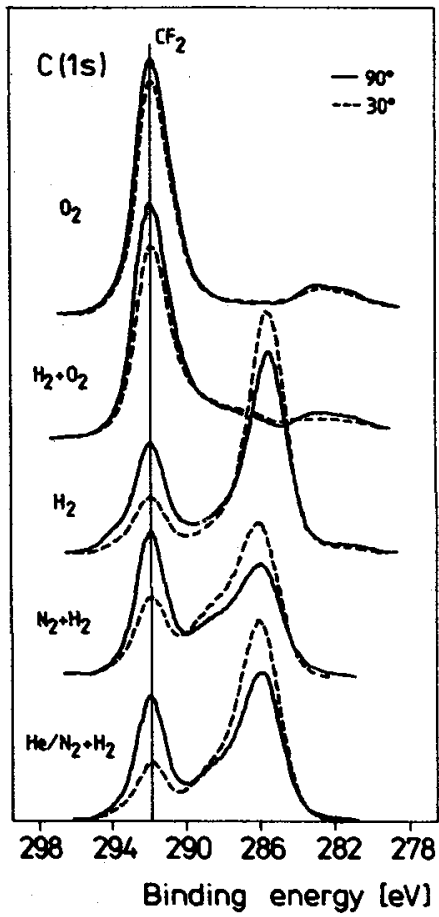

Figure 2: Comparaison des spectres $\mathrm{C}(1 \mathrm{~s})$ du Teflon PFA saisis à deux différents angles de sortie. Le traitement était effectué dans le plasma micro-onde, dans les gaz indiqués utilisant une pression de $200 \mathrm{~m}$ Torr et une durée d'exposition de $60 \mathrm{~s}$. (d'après réf. 19, utilisée avec la permission de John Wiley and Sons)

Dans notre laboratoire, nous utilisons la technique de microrayure pour évaluer l'adhérence [20]. Dans cette approche, une pointe hémisphérique de diamant se déplace le long d'une surface tandis que la force verticale est augmentée de façon linéaire pour déterminer la charge critique, $\mathrm{L}_{c}$, quand la couche (métal dans ce cas) commence de se délaminer. Les effets de différents gaz sur l'adhérence de Cu sur Teflon PFA sont trouvés à la figure 3. On constate que la meilleure adhérence est obtenue pour la surface traitée par $\mathrm{N}_{2}$, probablement due à la présence des liaisons fortes de $\mathrm{Cu}-\mathrm{N}-\mathrm{C}$. Ce dernier est supporté par les mesures XPS [21,22]. Il est quand même surprenant que l'augmentation de l'adhérence soit toujours moins importante lorsque l'hydrogène est présent. Ceci peut s'expliquer par le fait qu'une partie de l'hydrogène reste liée à la surface $(\mathbf{C}-\mathbf{H})$ et contribue à la formation d'une couche de faible adhérence. La présence de l'hydrogène à la surface était confirmée par les mesures par SSIMS [23]. On peut aussi noter à la figure 3 que l'adhérence est augmentée quand la surface de PFA est prétraitée par plasma de $\mathrm{He}$, suivie par l'exposition au plasma de $\mathrm{N}_{2}$. L'effet bénéfique de He est de stabiliser la surface par réticulation, dû à l'émission intensive de photons dans la région de l'ultraviolet lointain $(<100 \mathrm{~nm})$. 


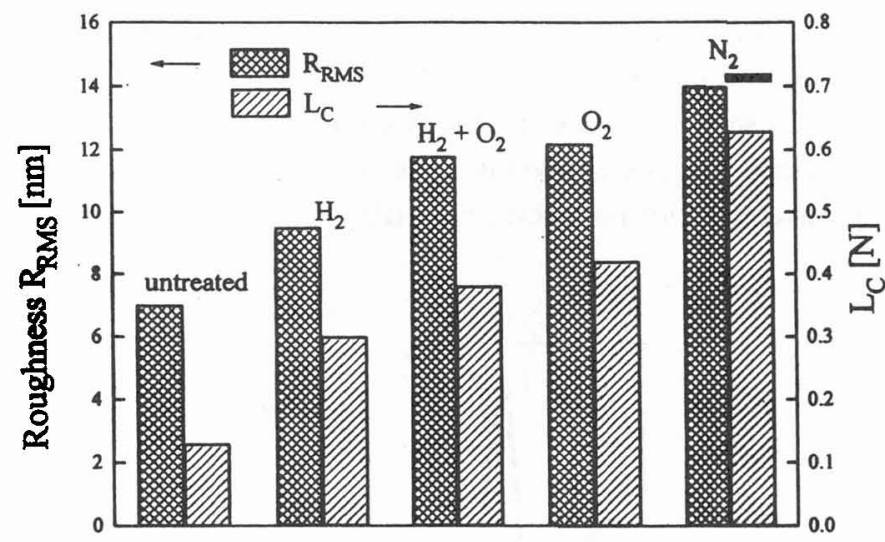

Treatment gas

Figure 3: Effet de traitement par plasma en présence de divers gaz sur les propriétés de surface du Teflon PFA: la rugosité moyenne $\left(R_{R M S}\right)$ mesurée par microscopie à force atomique et la charge critique $\left(L_{c}\right)$ pour $200 \mathrm{~nm}$ de Cu évaporé. Les traitements étaient effectués dans un plasma microonde, à une pression de $200 \mathrm{mT}$ Torr et une durée d'exposition de $60 \mathrm{~s}$. Pour $\mathrm{N}_{2}$, une amélioration additionnelle de l'adhérence est trouvée quand le plasma $\mathrm{N}_{2}$ est précédé par un plasma He. (d'après réf. 21, utilisée avec la permission de la Société Française du Vide)

Les phénomènes déjà discutés peuvent être expliqués par la présence d'une interphase structurée entre la couche métallique et le polymère, tel qu'illustrée à la figure 4 . Cette interphase consiste en une couche réticulée, densifiée et typiquement de quelques dizaines de $\mathrm{nm}$ d'épaisseur [24], suivie par une région modifiée chimiquement, préférablement menant aux liaisons de type métal-N-C. Une bonne adhérence requiert donc une optimisisation des paramètres de plasma afin d'effectuer le nettoyage de surface, la réticulation de l'interphase et la modification chimique. On devrait noter que l'adhérence optimale est reliée à la formation de liens chimiques du type couche- $\mathrm{N}$-substrat pour d'autres combinaisons de dépôts et de substrats, tel que l'Ag sur le polyethylène [25], le $\mathrm{Si}_{3} \mathrm{~N}_{4}$ sur le polycarbonate [26] et le $\mathrm{SiO}_{2}$ sur l'acier inoxydable [27]. Dans tous ces cas, l'augmentation de la microrugosité, telle que suggérée à la Figure 3, peut également contribuer à l'amélioration de l'adhérence, à la fois. En conséquence, ces réactions peuvent être généralisées pour d'autres types de polymères, tels que les fluoropolymères de faible $\kappa$ considérés pour les multicouches. Ces derniers comprennent le Teflon AF et les fluoropolymères plasma polymerisés et pulvérisés $[23,28]$. 


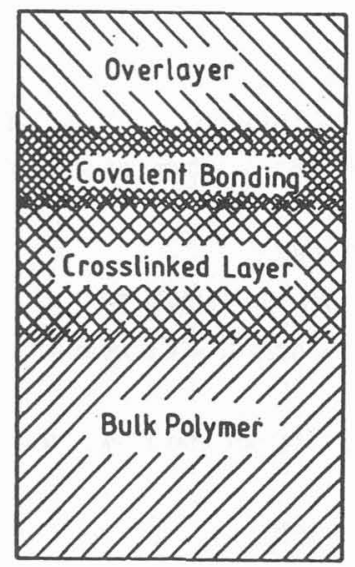

Figure 4: Schéma de l'interphase entre le dépôt et le substrat polymérique. (d'après réf. 11, utilisée avec la permission de VSP).

\section{CONCLUSION}

Nous avons discuté les problèmes associés à l'intégration des dispositifs multicouches basés sur le Cu et les fluoropolymères. En particulier, les réactions interfaciales sont faibles et les liens formés ne sont probablement pas suffisants pour maintenir la structure subissant des contraintes introduites par les inégalités des coefficients d'expansion thermique et hygroscopique des matériaux. Finalement, nous avons discuté de l'utilisation des plasmas pour modifier les surfaces polymériques pour améliorer l'adhérence.

\section{Remerciements}

Les auteurs remercient le Conseil de recherche en sciences naturelles et en génie du Canada et les Fonds pour la formation de chercheurs et l'aide à la recherche du Québec pour leur aide. 


\section{Références}

[1] S.P. Murarka, R.J. Gutmann, A.E. Kaloyeros et W.A. Lanford, Thin Solid Films 236 (1993) 257; ces temps de délai incluent aussi ceux associés au semiconducteur, et la géométrie et le type de dispositif.

[2] H. Oechsner, Z. Physik 238 (1970) 433.

[3] R.G. Hart et C.B. Cooper, Surf. Sci. 94 (1980) 105.

[4] A.J. Gordon et R.A. Ford, The Chemist's Companion, Wiley-Interscience, New York, 1972, p. 112.

[5] J.E. Huheey, Inorganic Chemistry (Harper and Row, New York, Second Edition, 1978) Appendix F.

[6] R.E. Honig, RCA Rev. 18 (1957) 195.

[7] Voir, par exemple, US Atomic Energy Commission Report ANL-5750, tel que trouvé dans le CRC Handbook of Chemistry and Physics (CRC Press, Cleveland, OH, 54 Édition, 1973-74) p. D-56.

[8] Voir, par exemple, E. Sacher, ACS Sympos. Series 440 (1990) 1 pour une liste de références.

[9] Voir, par exemple, J.-J. Pireaux, Ch. Grégoire, M. Vermeersch, P.A. Thiry, M. Rei Villar et R. Caudano, ACS Sympos. Series 440 (1990) 47 pour une liste de références.

[10] D. Popovici, J.E. Klemberg-Sapieha, G. Czeremuszkin, E. Sacher, M. Meunier et L. Martinu, Microelectron. Eng., à paraître.

[11] E.M. Liston, L. Martinu et M.R. Wertheimer, J. Adhesion Sci. Technol. 7 (1993) 1091.

[12] S. Novak et O.M. Küttel, dans "Plasma Properties, Deposition and Etching" J. Pauch and S.A. Alterowitz, eds. (Trans. Tech. Publications, Aedermensdorf 1994), p. 705.

[13] F.D. Egitto et L.J. Matienzo, IBM J. Res. Develop. 38 (1994) 423.

[14] M.R. Wertheimer, L. Martinu et E.M. Liston, dans "Handbook of Thin Film Process Technology" (IOP Publishing, Bristol 1996) Chapter E3.0.

[15] K.L. Mittal, J. Vac. Sci. Technol. 13 (1976) 19.

[16] L.H. Sharpe, dans "The Interfacial Interactions in Polymeric Composites", G. Akovali, ed. (NATO-ASI Ser. E: Applied Sciences, Kluwer, Dordrecht 1994) p. 230.

[17] "Techniques and Applications of Plasma Chemistry", J.R. Hollahan and A. Bell, eds. (Wiley, New York, 1974).

[18] "Plasma Deposition, Treatment and Etching of Polymers", R. d'Agostino, ed. (Academic Press, Boston, 1990).

[19] M.K. Shi, L. Martinu, E. Sacher, A. Selmani, M.R. Wertheimer et A. Yelon, Surf. Interface Anal. 23 (1995) 99.

[20] P, Leroux, A. Raveh, J.E. Klemberg-Sapieha et L. Martinu, dans "Proc. 36th Ann. Tech. Conf." (Soc. Vac. Coaters, Albuquerque, 1993), p. 472.

[21] J.E. Klemberg-Sapieha, M.K. Shi, L. Martinu et M.R. Wertheimer, dans "CIP '95" (Société Française du Vide, 1995), p. 151; J.E. Klemberg-Sapieha, dans "Plasmas and Polymers", R. d'Agostino, ed., (Kluwer Academic Publishers, Boston, a paraître).

[22] M.K. Shi, A. Selmani, L. Martinu, E. Sacher, M.R. Wertheimer et A. Yelon, J. Adh. Sci. Technọl. 8 (1994), 1129.

[23] M.K. Shi, J.E. Klemberg-Sapieha, G. Czeremuszkin, L. Martinu et E. Sacher, dans "Proc. ISPC12" (ISPC, Minneapolis, 1995), p. 51. 
[24] S. Vallon, B. Drévillon, F. Poncin-Épaillard, J.E. Klemberg-Sapieha et L. Martinu, J. Vac. Sci. Technol. A, 14 (1996) 3194.

[25] L. Gerenser, J. Vac. Sci. Technol. A, 6 (1988) 2897.

[26] J.E. Klemberg-Sapieha, D. Poitras, L. Martinu, N.L.S. Yamasaki et C.W. Lantman, J. Vac. Sci. Technol. $A$, à paraître.

[27] N. Bertrand, B. Drévillon, J.E. Klemberg-Sapieha et L. Martinu, Thin Solid Films, à paraître.

[28] A. Alptekin, E. Sacher, G. Czeremuszkin, L. Martinu et M. Meunier; dans "MRS Symp. Proc. Low Dielectric Constant Materials", (MRS, Pittsburg, 1997), à paraître. 